\title{
Acute Effects of Whole Body Vibration on Shoulder Muscular Strength and Joint Position Sense
}

\author{
by \\ Junggi Hong ${ }^{1}$, Mayachela T. Velez', Abraham M. Moland ${ }^{1}$, Jeff A. Sullivan ${ }^{2}$
}

\begin{abstract}
Functional changes following whole body vibration (WBV) training have been attributed to adaptations in the neuromuscular system. However, these changes have mainly been observed in the lower extremity with minimal change to the upper extremity. The purpose of the study is to examine the acute effect of shoulder vibration on joint position sense and selected muscle performance characteristics (peak torque, time to peak torque, and power). Forty young individuals (19.84 $\pm 1.73 \mathrm{yrs}, 171.41 \pm 7.73 \mathrm{~cm}, 70.07 \pm 9.32 \mathrm{~kg}$ ) with no history of upper body injuries were randomly assigned to an experimental (Vibration) or control (No-Vibration) group. To assess shoulder proprioception, active and passive joint position senses were measured on both internal and external rotation of the shoulder. The muscle performance variables (peak torque and time to peak torque) were measured using isokinetic dynamometer with the velocity of $60 \% \mathrm{sec}$. After three bouts of 1 minute vibration training, the experimental group demonstrated a significant improvement in the internal rotation peak torque, time to peak torque and external rotation time to peak torque $(p<0.05)$. However, no-significant differences were revealed for joint position sense, external rotation peak torque, and time to peak torque between the groups. Our findings suggest that short bouts of vibration treatment have a significant effect on shoulder muscle characteristics.
\end{abstract}

Key words: whole body vibration, joint position sense, proprioception, peak torque

\section{Introduction}

The glenohumeral joint's relatively poor osseous and capsuloligamentous stability necessitates a reliance on neuromuscular proprioception and stabilization more than any other joints in the human body (Barden et al., 2004). Proprioception refers to the integration of internal sensory information pertaining to joint position, muscle tension and location of the body part in space (Gooey et al., 2000). Proprioception contributes to the motor programming for neuromuscular control required for dynamic joint stability and also contributes to precision movements.
Both dynamic stability of the shoulder joint and fine coordination of multi-joint movement sequences require rapid and accurate afferent input from the receptors around the shoulder joints and muscles involved. A loss of proprioception would contribute to neuromuscular dysfunction and likely poor segmental stability in the risk of injury. For example, numerous studies have shown that trauma to tissues that contain proprioceptors result in partial deafferentation and major proprioceptive deficits in movement control, which result in increased movements variability and the inability to maintain static postures. (Myers and Lephart, 2002; Lephart and Fu, 2000). Therefore, use of exercises designed to main-

\footnotetext{
- Department of Exercise Science at Willamette University, Oregon, USA

2 - Point Loma Nazarene University
} 
tain and improve the integrity and functional stability of the shoulder joint is considered to be an important element of most training and rehabilitation training process.

Recently, whole body vibration (WBV) training has become increasingly used in sports training and injury treatment settings as an efficient neuromuscular training tool. A previous study suggests WBV exercises can not only enhance acute strength and power capabilities, but also improve proprioception (Fontana et al., 2002). For example, Fontana et al. (2002) reported that adding whole-body vibration to a simple weight-bearing exercise enhances lumbosacral position sense after a single 5-minute WBV session. Researchers have postulated that vibration provides the sensorimotor system with additional afferent input that may facilitate position sense (Bosco et al., 1999; Cardinale and Lim, 2003; Roelants et al., 2004; Torvinen et al., 2002 ).

Several mechanisms for the acute effects of WBV training have been suggested, including neural adaptation, related to increased muscle activation, caused by increased excitability input from muscle spindles exposed to a vibration (Abercromby et al., 2007; Cardinale and Bosco, 2003). It has been hypothesized that the enhanced muscle power observed following acute vibration occurs via tonic vibration reflex (TVR), whereby stimulation of muscle spindles (Ia afferents) results in reflex activation of motoneurones with increased spatial recruitment (Bishop et al., 1974; Bongiovanni and Hagbarth, 1990). It has also been suggested that the increased level of neural drive following vibration training is closely related to increased muscle stiffness, which is known to contribute to joint position sensibility (Fontana et al., 2005; Jerosch and Prymka, 1996)

Based on the theoretical mechanism of vibration training proposed by researchers, it is likely that increased neural drive and muscle stiffness induced by WBV would enhance not only the shoulder muscular function but also shoulder propiroception. However, the lack of standardization of vibration training protocols and the lower extremities-focused studies, make it difficult to completely understand the underlying mechanisms of how vibration training may affect upper body proprioception and muscle function (Bosco et al., 1999; Cardinale and Lim, 2003; Roelants et al., 2004; Torvinen et al., 2002). Therefore, the purpose of this study was to investigate the acute effects of whole body vibration on muscular function and joint position sense of the shoulder joint in

\begin{tabular}{|lcc|}
\hline & \multicolumn{2}{c|}{ Table 1 } \\
\hline & Participants' $^{\prime}$ demographics \\
\hline Mumber of participants & Male & Female \\
Age (yrs) & 22 & 18 \\
Height $(\mathrm{cm})$ & $19.45 \pm 1.78$ & $20.23 \pm 1.69$ \\
Weight $(\mathrm{kg})$ & $175.68 \pm 9.21$ & $167.14 \pm 6.24$ \\
\hline \multicolumn{3}{r}{} \\
\hline
\end{tabular}

young healthy individuals. We hypothesized that subjects in the vibration group would have increased muscle function and joint position sense (JPS) following acute exposure to vibration treatment.

\section{Methods}

Forty (18 female and 22 male) healthy individuals, between the ages of 18 and 35, participated in the study from Willamette University and the surrounding community (Table 1 ). Participants were excluded from the study if they had experienced a shoulder injury within the 12 months prior to the study. The protocol was approved by the Willamette University Institution Review Board, and all participants gave written informed consent prior to engaging in any study activities.

\section{Joint Position Sense (JPS) Testing}

Glenohumeral joint position sense (JPS) was assessed with an isokinetic dynamometer (Biodex MultiJoint System 3 Pro, Biodex Medical, Inc., Shirley, NY) by employing a passive reproduction of passive positioning and active reproduction of passive positioning. A custom-designed shoulder positioning device secured the subject's limb to the dynamometer. Prior to testing, each subject's maximal passive external rotation range of motion (ERmax) of the dominant arm was measured while seated in the dynamometer's chair, with the shoulder in 90 degree abduction and neutral rotation. Two relative target angles were then calculated to evaluate JPS for each subject, based on $60 \%$ and $90 \%$ of the ERmax value of the dominat shoulder. Two previous studies have used a joint position sense testing protocol similar to the present study's to establish target angles (Janwatanankul et al., 2001; Swanik et al., 2002).

Three practice trials were performed on the dominant limb to acquaint subjects with the JPS testing protocol. To begin testing, subjects were blindfolded while seated on the dynamometer, with their shoulder in a position of function in the frontal plane (90 degree of shoulder abduction, and elbow flexion in neutral rotation (Figure 1)). To minimize 


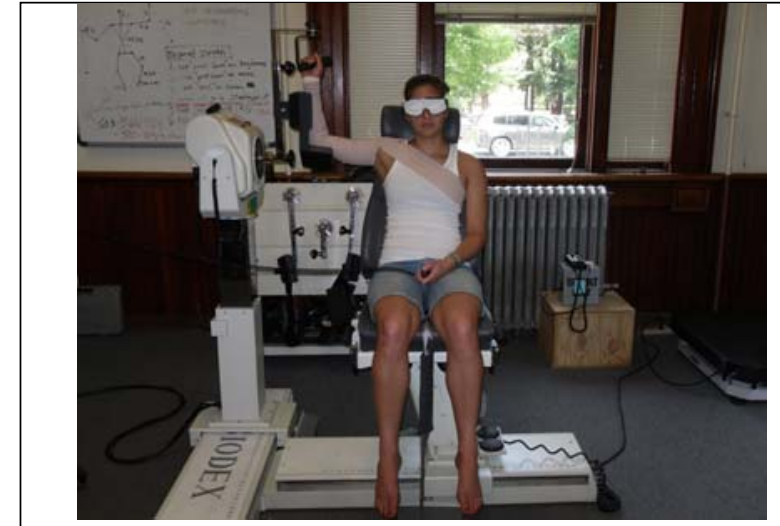

Figure 1

Joint position sense testing position

sensory input from cutaneous receptors, a 5" wide stockinette was placed over the subject's arm, and an upper-extremity vacuum splint (Cramer Products, Inc., Gardener, Kansas) was applied and inflated evenly over the stockinette covering the fingers to the mid-brachium. The splinted limb was then secured to the dynamometer's lever arm with a 6" elastic bandage. After a standardized warm-up acquainting the subject with the dynamometer, the continuous passive motion (CPM) mode of the dynamometer was employed for JPS testing.

The shoulder under investigation was passively rotated by the dynamometer at a velocity of $5 \% \mathrm{sec}$ from neutral rotation to one of the two target angles, $60 \%$ ERmax or $90 \%$ ERmax. The order of JPS testing was counterbalanced between target positions to control for bias caused by learning and/or testing fatigue. The target position was maintained for $10 \mathrm{sec}-$ onds with subjects verbally instructed to "focus on the position of their shoulder." The arm was then passively returned to the starting position at $30 \% \mathrm{sec}$ and rested for 5 seconds. The dynamometer then was activated to passively move the shoulder into external rotation at the initial velocity of $5 \%$ sec. Subjects were instructed to identify the target angle by pressing the dynamometer's hand-held cutoff switch to stop the dynamometer when they perceived that the target position had been reached. A total of 6 successful trials were performed on the shoulder ( 3 trials at $60 \%$ ERmax, 3 trials at $90 \%$ ERmax)

To test active JPS, the shoulder under investigation was passively rotated by the dynamometer at a velocity of $5 \% \mathrm{sec}$ from neutral rotation to one of the two target angles, $60 \%$ ERmax or $90 \%$ ERmax. The target position was maintained for 10 seconds with subjects verbally instructed to "focus on the position of their shoulder." The arm was then passively re-
Table 2

Joint position sense testing protocols: a different protocol was chosen for each subject to control for bias caused by learning and/or testing fatigue

\begin{tabular}{ccccccc}
\hline \multicolumn{7}{c}{ JPS trials } \\
& 1 & 2 & 3 & 4 & 5 & 6 \\
\hline Protocol 1 & $60 \%$ & $90 \%$ & $60 \%$ & $90 \%$ & $60 \%$ & $90 \%$ \\
Protocol 2 & $90 \%$ & $60 \%$ & $90 \%$ & $60 \%$ & $90 \%$ & $60 \%$ \\
Protocol 3 & $60 \%$ & $60 \%$ & $90 \%$ & $60 \%$ & $90 \%$ & $90 \%$ \\
Protocol 4 & $90 \%$ & $90 \%$ & $60 \%$ & $90 \%$ & $60 \%$ & $60 \%$ \\
\hline
\end{tabular}

turned to the starting position at $30 \% \mathrm{sec}$ and rested for 5 seconds. Each subject was then asked to actively reposition the shoulder and to "stop" when he or she felt that the previous target angle had been reached (Sullivan, Hoffman and Harter, 2008). A total of 6 trials were performed on the same shoulder. The angle at which the subject stopped was recorded and subtracted from the initial preset angle. An average of the absolute value of the 12 errors was used for statistical analysis. The order of testing was counter-balanced for each subject (Table 2).

\section{Glenohumeral Muscular Strength Testing}

After joint position sense testing, subjects were seated in the chair of the isokinetic device in a resting position with the arm in $90^{\circ}$ abduction and $90^{\circ}$ elbow flexion with neutral rotation in the scapular plane (Figure 1). Concentric internal rotation (IR) and external rotation (ER) peak torques were measured using 5 maximal testing repetitions at $60^{\circ} / \mathrm{sec}$.

To orient subjects with the dynamometer testing protocol, 5 sub-maximal repetitions of IR/ER were performed at $60^{\circ} / \mathrm{sec}$. The dynamometer's gravity correction software feature was employed as gravity could potentially influence the internal rotation and external rotation strength values. The single highest peak torque value obtained from 5 maximal repetitions was used for statistical analysis.

\section{Shoulder Vibration Training}

Subjects were sex stratified and assigned to either a Vibration $(n=19)$ or No-Vibration group $(n=21)$. Once baseline testing had been completed, participants were instructed to place their arms shoulder width apart on the vibration platform in a plank position, with their feet shoulder width apart on the ground. Researchers demonstrated the correct position to prevent participants from arching their backs or raising their gluteals (Figure 2). Participants were instructed to hold this position for 1 minute for three 


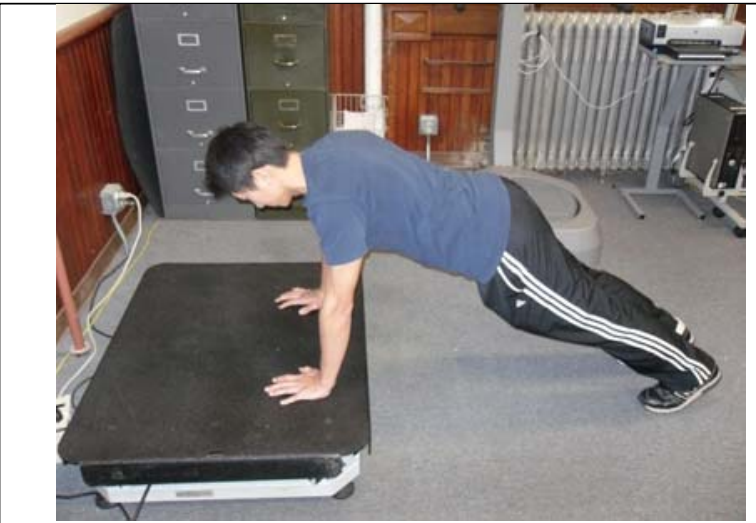

Figure 2

Experimental set up for vibration training in a push up position

sets, with a minute rest in between. Those in the treatment group experienced vertical vibration at an amplitude of $5 \mathrm{~mm}$ and at a frequency of $30 \mathrm{~Hz}$. Those in the control group maintained the same position for the same period of time without vibration. After the intervention, the same testing protocol from baseline testing was applied and the differences between the results analyzed.

\section{Statistical Analysis}

All statistical analyses were performed using the SPSS 16.0 software (SPSS, Inc., Chicago, IL). To assess the effects of vibration training on shoulder isokinetic measures and joint position sense, 2 (group) x 2 (time) mixed design ANOVA was used for each variable. Alpha was set at 0.05.

\section{Results}

\section{Joint position sense}

The overall data for the JPS testing is shown in Table 3. After acute bouts of shoulder vibration exposure, results revealed no significant differences between the vibration and No-Vibration groups in measurement for active reproduction of passive positioning at the reference of both $30 \%$ and $90 \%$ of external rotation ( $p=0.431$ and $p=0.792$, respectively). For passive reproduction of passive positioning, there were also no significant differences revealed at either $30 \%$ or $90 \%$ of external rotation for all subjects ( $p=0.881$ and $p=0.525$, respectively).

The maximal isokinetic muscular strength, as measured by the shoulder internal and external rotation of the dominant arm, is shown in Table 4. After short vibration exposure, results revealed signifi-

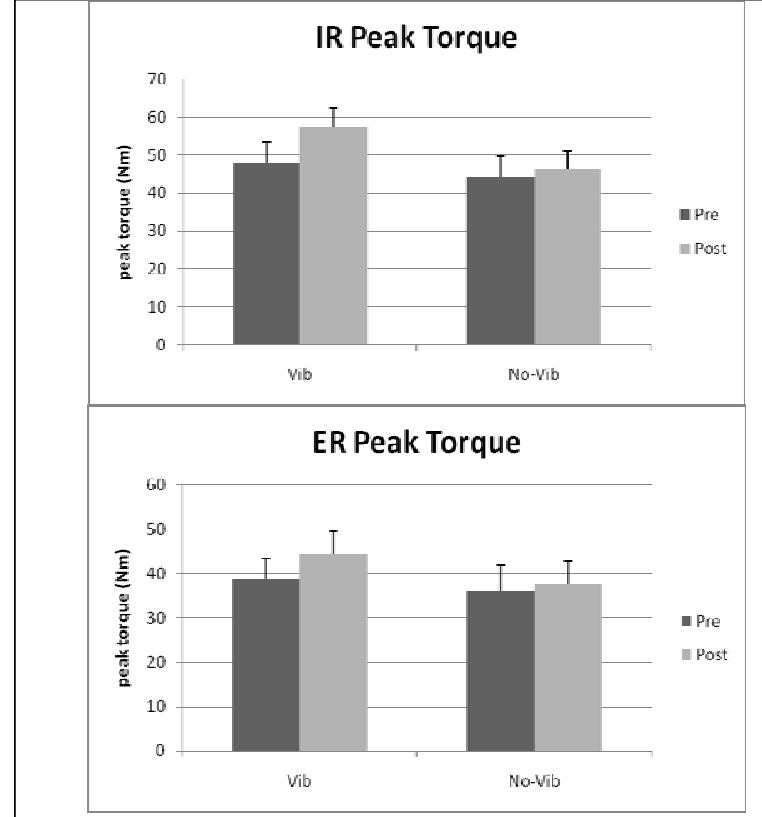

Figure 3

Changes in glenohumeral IR and ER peak torque after WBV
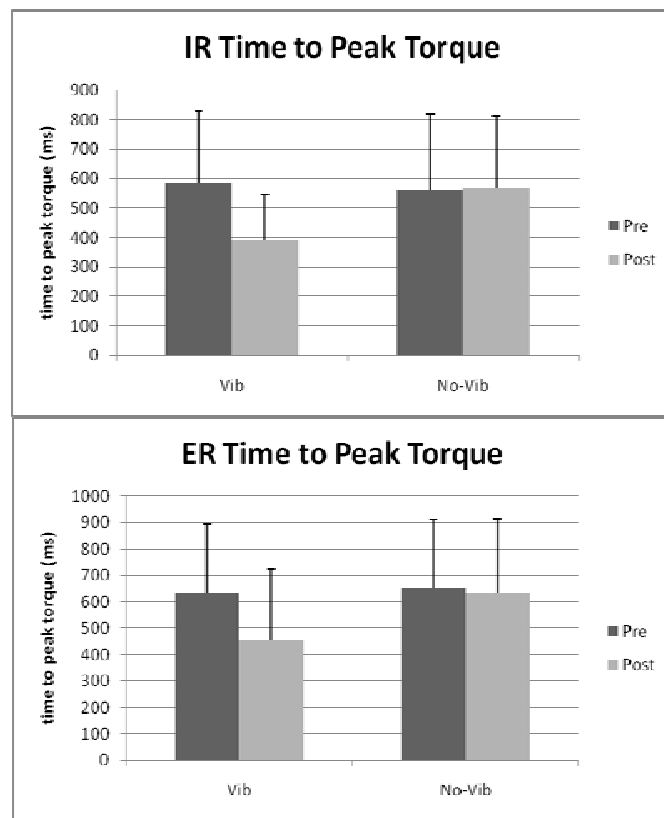

Figure 4

Changes in glenohumeral IR and ER time to peak torque after $W B V$

cant group differences for internal rotation peak torque $(p=0.042)$. External rotation peak torque improved by $13.5 \%$ following treatment in the vibration group, although the change was not statistically significant $(p=0.058)$. The No-Vibration group did not show any significant changes in peak torque of IR and ER ( $p>.05)$ (Figure 3).

The results revealed significant group differences for time to peak torque for both internal and external 


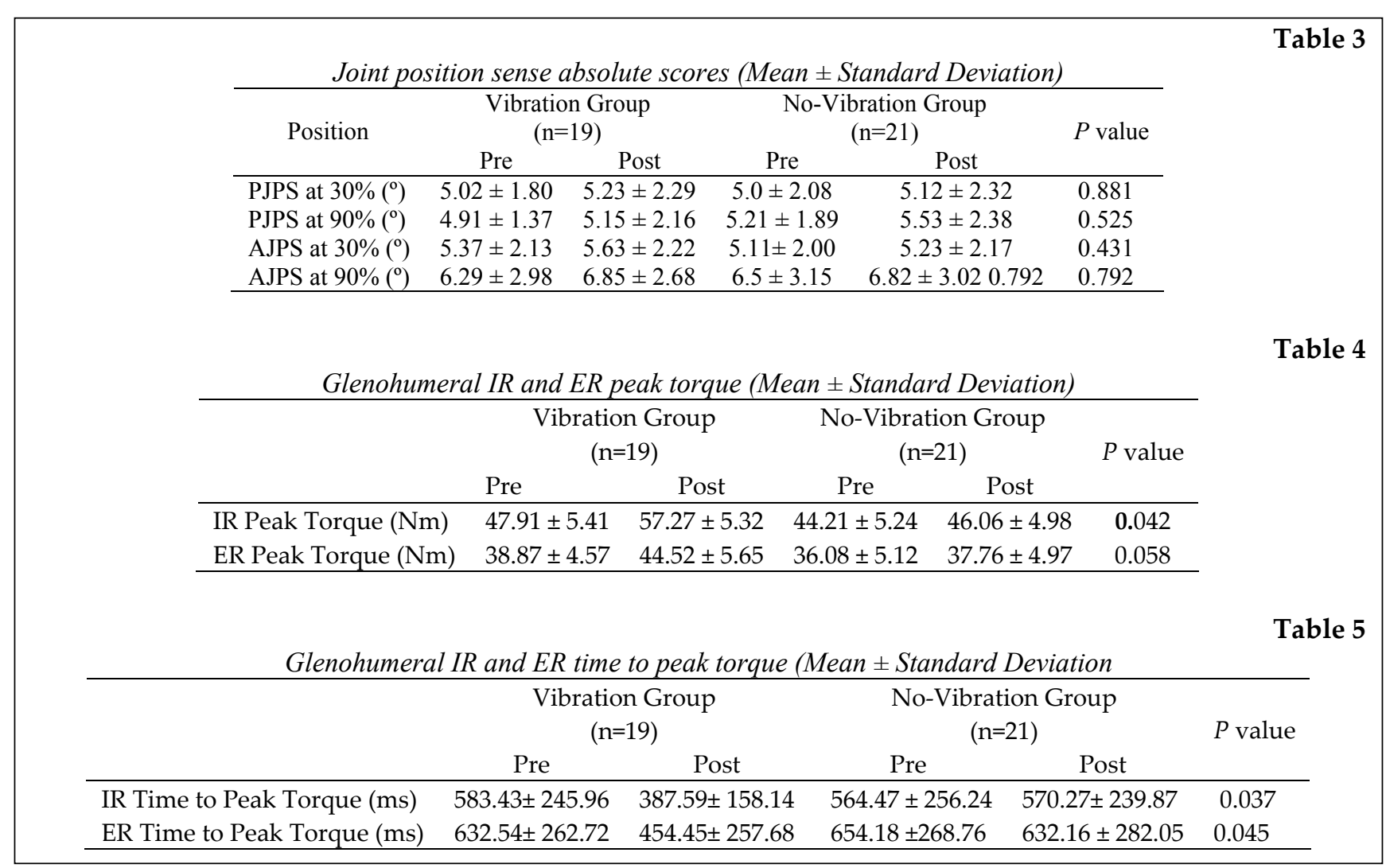

rotation ( $p=0.037$ and $p=0.045$, respectively). The NoVibration group did not show any notable changes for time to peak torque of IR and ER between preand post-testing $(p>0.05)$ (Figure 4$)$.

\section{Discussion}

The primary finding of the study was that three bouts of one-minute vibration treatment $(30 \mathrm{~Hz}$ and 5 $\mathrm{mm}$ ) resulted in significant improvements in both shoulder internal rotation peak torque, time to peak torque, and external rotation time to peak torque $(p<0.05)$. The external rotation peak torque improved by $13 \%$ after the vibration treatment, however, the data analysis revealed the improvement of peak torque was statistically not significant $(p=0.058)$. Although statistical significance was not detected on ER peak torque, there was a tendency towards significance with increased muscle activity, as seen in the IR peak torque. The results observed in the present study are consistent with the findings demonstrated in previous studies. Both Rittweger et al. (2003) and Ruiter et al. (2003) have demonstrated acute effects of WBV on muscular performance, as measured with vertical jump, maximal isometric force and maximal force rise time. Rittweger et al. (2003) assessed jump height, ground contact time, and tendon reflex before and after WBV stimulation in 19 healthy young volunteers (Rittweger et al., 2003). The study showed that the subjects who performed the squatting exercise on the vibration platform had greater tendon reflex amplitude and vastus lateralis mean frequency during isometric torque. These authors suggested that superimposed vibration appears to elicit an alteration in neuromuscular recruitment patterns, which apparently enhance neuromuscular excitability (Rittweger et al., 2003; De Ruiter et al., 2003).

Regarding the acute effects of vibration training on the upper extremity, a study by Bosco et al. (1999) investigated the effects of vibration training on upper arm muscle, and found that electromyography of the elbow flexors showed increased neural activity with the vibration stimulus, which was more than twice the baseline values. (Bosco et al., 1999). Although most of these studies have focused on the lower extremity, the improvements in the shoulder muscle function observed in our study can be attributed to an increased enhancement of neuromuscular excitability (Wilcock et al., 2009). Theoretically, vibration causes the afferent neurons from the muscle spindles to become more sensitive to muscle stretch, which increases the activation of alpha motor neurons in response. This response may lead to increased motor unit recruitment, increased firing frequency and/or improved muscular contraction, al- 
lowing a quicker and more forceful muscular contraction when the muscle is rapidly stretched (Bosco et al., 1999; Wilcock et al., 2009).

Another reason for the increased isokinetic peak torque and time to peak torque shown in the present study can be found in the connection between the roles of pre-activation and muscle stiffness (Riemann et al., 2002). Pre-activation and muscle stiffness are often addressed at the knee and ankle joints (McNair et al., 1992). In our study, the subjects in the Vibration group held the push up position for three bouts of 1 minute. Both arms were directly positioned on the vibration platform and the subjects were asked to hold the position while the vibration was applied. The No-Vibration group assumed the same position for the same amount of time. In order to hold this position, the subjects were isometrically contracting their arms and the shoulders, either against their body weight and gravity, or vibration. The lack of changes in isokinetic measures in the No-Vibration group suggest that the push up position without vibration, for three bouts of 1 minute, did not induce any increased muscle activity. With regard to the effects of isomeric contraction with vibration, Abercromby et al. (2007) demonstrated greater strength gains during isometric contractions, combined with acute vibration exposure. According to Roelants et al. (2006b), vibration effect can be larger in the posture in which the receptor-bearing muscle was more pre-activated (Roelants et al., 2006b). In addition, it was shown that even a small increase in pre-activation may lead to increased muscle spindle sensitivity because of alpha-gamma co-activation, which was known to increase muscle stiffness (Burke et al., 1976). In this respect, we believe that the push up position held during vibration treatment might have increased the level of pre-activation of the muscles around the shoulder and resulted in increased muscle strength.

We hypothesized that acute vibration exposure to the shoulder joint would improve both passive and active joint position sense. The rationale for our hypothesis was based on the theoretical mechanisms suggesting that vibration would have an effect, not only on the musculotendinous structures, but also on the joint structures, which indicates additional potent sensory motor effects through the proprioceptive joint mechanoreceptors (Roll et al., 1980). In this respect, we examined both passive reproduction of passive positioning (PRPP) and active reproduction of passive positioning (ARPP) as our joint position sense testing variables. In theory, PRPP is known to receive JPS input mainly from capsuloligamentous receptors, and ARPP is more reliant on muscle receptors (muscle spindles and golgi tendon organs) (Gross, 1987; Myers et al., 2000).

Contrary to our hypothesis, the results of the study did not reveal a significant training effect on absolute errors at the two different target angles of AJPS, or at the two different target angles of PJPS $(p>0.05)$. These results contradict previous studies that recently demonstrated positive effects of vibration training on joint position sense (Fontana et al., 2002; Tripp et al., 2009). Fontana et al. (2002) reported that adding whole body vibration to a simple weight-bearing exercise enhances lumbosacral position sense after a single 5-minute session. Although the vibration stimulus in this study was a handheld vibration, which is a different form of vibration compared to most recent vibration studies, another study by Tripp et al. (2009) reported that $15 \mathrm{~Hz}$ vibration enhanced accuracy and decreased variability of elbow joint position sense. Regarding the responsible mechanisms for such positive effects, the authors have postulated that vibration provides the sensorimotor system with additional afferent input that may facilitate joint position sense. It has been also suggested that vibration increases joint stiffness by activation of joint mechanoreceptors and stimulation of the gamma efferents, which is known to be closely associated with enhanced joint position sense (Fontana et al., 2002).

Despite the contradicting results to earlier studies, a possible explanation for the unchanged joint position sense observed in our study can be found in the findings of a recent study by Radovanovic et al. (1998, 2002). According to the authors, vibration-induced alternations in proprioceptive messages result in disturbances of both position and movement senses (Eklund and Hagbarth, 1965; Eklund, 1972; Goodwin et al., 1972; Jaric and Corcos, 1994). Furthermore, this erroneous kinaesthetic information, derived from vibration-induced afferent inflow from muscle spindles, affects the performance of both slow and fast voluntary movements (Capaday et al., 1981; Latash, 1993; Sittig et al., 1985). Since the variation in form of vibration and protocols used between our study and other studies suggest negative effects of vibration on JPS, a direct comparison and further elaboration does not possible at this point. However, to better understand the mechanisms by which vibration may enhance JPS acuity, further investigation is warranted. 
As in all research, our study had limitations and identified areas that warrant additional investigation. A larger sample size may have helped to elucidate the association between vibration training and muscular function. The present study only investigated the acute effects of vibration training, however, chronic effects of vibration on muscle function and joint position sense in the upper extremity need to be examined as well. In future studies, researchers should include electromyography (EMG) or mechanomyography (MMG) to better examine any electrical and mechanical changes in the muscle during or after vibration training. It is also recommended that researchers include clinical populations to investigate any implications for vibration training as a possible therapeutic aid.

\section{Conclusion}

The study observed acute improvements in shoulder internal rotation peak torque, time to peak torque and external rotation time to peak torque after three bouts of 1 minute vibration exposure. However, vibration training didn't appear to have an effect on either passive or active shoulder joint position sense. The findings here may provide a means of isolating the effect of WBV on the neuromuscular system of the upper body, and perhaps may help researchers involved in the health, fitness and therapeutic fields to better understand and determine the potential of WBV as an efficient interventional tool for rehabilitation and training. Further research is needed to investigate other mechanisms that may underlie the physiological responses and adaptation to WBV, and how these responses may occur among individuals with abnormal neuromuscular function.

\section{References}

Abercromby AF, Amonette WE, Layne CS, McFarlin BK, Hinman MR, Paloski WH. Variation in neuromuscular responses during acute whole-body vibration exercise. Med Sci Sport Exer 2007. 39:1642-1650.

Bishop, B. Vibratory Stimulation: Part 1. Neurophysiology of Motor Responses Evoked by Vibratory Stimulation. Physical Therapy 1974.54:1273-1282.

Bongiovanni LG. and Hagbarth KE. Tonic vibration reflexes elicited during fatigue from maximal voluntary contractions in man. J Physiol 1990. 423:1-14.

Bosco C, Cardinale M, Tsarpela O. Influence of vibration on mechanical power and electromyogram activity in human arm flexor muscles. Eur J Appl Physiol O 1999. 79(4):306-311

Bosco C, Colli R, Introini E, Cardinale M, Tsarpela E Adaptive responses of human skeletal muscle to vibrations exposure. Clin Physiol 1999. 19:183-187.

Barden JM, Balyk R, Raso J, Mareau M, Bagnail K Dynamic upper limb proprioception in multidirectional shoulder instability. Clinical Orthopedics Related Research 2004. 420:181-189.

Burke D, Hagbarth KE, Lofstedt L, and Wallin BG. The responses of human muscle spindle endings to vibration of non-contracting muscles. J Physiol 1976. 261:673-693.

Capaday C, Cooke JD. The effects of muscle vibration on the attainment of intended final position during voluntary human arm movement. Exp Brain Res 1981. 42:228-230.

Cardinale, M., Bosco, B. The use of vibration as an exercise intervention. Med Sci Sport Exer 2003. 31:3-7.

Cardinale M, Lim J. The acute effects of two different whole body vibration frequencies on vertical jump performance. Medicine and Sport Science 2003. 56:287-292.

De Ruiter C, Van der Linden R, Van der Ziden A, Hollander P, De Haan A. Short-term effects of whole body vibration on maximal voluntary isomeric knee extensor force and rate of force rise. Eur J App Physiol 2003. $88: 472-475$

Eklund G. Position sense and state of contraction; the effects of vibration. J Neurol Neurosur Ps 1972. 35:606-611.

Eklund, G., Hagbarth, KE. Motor effects of vibratory muscle stimuli in man. Electroencephalography and Clinical Neurophysiology. 1965. 19:619. 
Fontana TL, Richardson CA, Stanton WR. The effect of weight-bearing exercise with low frequency, whole body vibration on lumbosacral proprioception: A pilot study on normal subjects. Aust J Physiother 2002. 51:259-263.

Goodwin GM, McCloskey DI, Matthews PBC. The contribution of muscle afferents to kinaesthesia shown by vibration induced illusions of movement and by the effects of paralyzing joint afferents. Brain 1972. 95:705-748.

Gooey K. Bradfiled O, Talbot J, Morgan DL, Proske U. Effects of body orientation, load and vibration on sensing position and movement at the human elbow joint. Exp Brain Res 2000. 133:340-348.

Gross MT. Effects of recurrent lateral ankle sprains on active and passive judgments of joint position. Phys Ther 1987. 67:1505-1509.

Hand J, Verscheure S, Osternig L. A comparison of whole body vibration and resistance training on total work in the rotator cuff. J Athl Training 2009. 44(5):469-474.

Janwantanakul P, Jones MA, Magarey ME, Miles TS, Grimmer KA. Characteristics of shoulder position sense: effects of mode of movement, scapular support, and arm orientation. J Sport Rehabil 2001. 11(3):157-168

Jaric S, Corcos DM, Gottlieb GL, Ilic DB, Latash ML The effects of practice on movement distance and final position reproduction: implication for the equilibrium-point control of movements. Exp Brain Res 1994. 100:353-359.

Jerosch K, Prymka M. Proprioception and joint stability. Knee Surgery Sports Trumatology Arthroscopic 1996. 4:171-179.

Latash ML. Control of human movement. Urbana, Human Kinetics 1993.

Lephart SM, Fu FH Proprioception and Neuromuscular Control in Joint Stability. Champaign, Human Kinetics. 2000

McNair PJ, Wood GA, Marshall RN. Stiffness of the hamstring muscles and its relationship to function in anterior cruciate deficient individuals. Clin Biomech 1992. 7:131-137.

Myers JB, Lephart SM. Sensorimotor deficits contributing to glenohumeral instability. Clin Orthop Relat R 2002. 400:98-104.

Myers JB, Lephart SM, Bradley JP, Riemann EL, Fu FH. Evaluation of shoulder proprioception and function following thermal capsulorrhaphy. Med Sci Sport Exer 200032:S123.

Radovanovic S, Jaric S, Milanovic S, Vukcevic I, Ljubisavljevic M, Anastasijevic R. The effects of prior antagonist muscle vibration on performance of rapid movements. J Electromyogr Kines 1998. 8:139-145.

Radovanovic S, Korotkov A, Ljubisavljevic M, Lyskov E, Thunberg J, Kataeva G, Danko S, Roudas M, Pakhomov S, Medvedev S, Johansson H. Comparison of brain activity during different types of proprioceptive inputs: a positron emission tomography study. Exp Brain Res 2002. 143: 276-285.

Riemann B, Myers BL, Lephart SM. Sensorimotor system measurement techniques. J Athl Training 2002. 37(1):8598.

Rittweger J, Mutschelknauss M, Felsenberg D. Acute changes in neuromuscular excitability after exhaustive whole body vibration exercise as complared to exhaustion by squatting exercise. Clinical Physiology and Functional Imaging 2003. 23:81-86.

Roelants, M., C. Delecluse, and Verschueren S. M. Whole-Body-Vibration Training Increases Knee-Extension Strength and Speed of Movement in Older Women. Journal of the American Geriatrics Society 2004. 52:901-908.

Roelants M, Verschueren S, Delecluse C. Whole-body-vibration-induced increase in leg muscle activity during different squat exercises J Strength Cond Res 2006. 20(1):124-129.

Rogers D, Bendrups A, Lewis M. Disturbed proprioception following a period of muscle vibration in humans. Neuroscience Letter 1985. 57:147-152. 
Roll JP, Martin B, Gauthier GM. Effects of whole body vibration on spinal reflexes in man. Aviat Space Envir Md 1980. 51:1227-1233.

Sittig AC, Van Der Gon JJD, Gielen CCAM, Van Wijk AJM. The attainment of target position during step tracking movements despite a shift of initial position. Exp Brain Res 1985. 60:407-410.

Sullivan JA, Hoffman MA, Harter RA Shoulder joint position sense after thermal, open, and arthroscopic capsulorrhaphy for recurrent anterior instability. Journal of Shoulder and Elbow Surgery 2008. 17(3):389-394.

Swanik KA, Lephart SM, Swanik CB, Lephart SP, Stone DA, Fu FH. The effects of shoulder playometric training on proprioception and selected muscle performance characteristics. Journal of Elbow Surgery 2002. 11(6):579586.

Torvinen, S., H. Sievanen, T. A. Jarvinen, M. Pasanen, S. Kontulainen, and Kannus P. Effect of 4-min vertical whole body vibration on muscle performance and body balance: a randomized cross-over study. Int J Sports Med 2002. 23:374-379.

Tripp BL, Faust D, Jacobs P. Elbow joint position sense after neuromuscular training with handheld vibration. J Athl Training 2009. 44(6):617-623.

Wilcock IM, Whatman C, Harris N, Keogh J. Vibration training: could it enhance the strength, power, or speed of athletes? J Strength Cond Res 2009. 23(2):593-603.

\section{Acknowledgements}

This work was supported by Mary Stuart Rogers Scholarship Fund. The authors are grateful for the support and insights provided by Dr. Jeff Sullivan for the study.

\section{Corresponding author}

\section{Junggi Hong, Ph.D, ATC}

Assistant Professor of Department of Exercise Science at Willamette University

900 State Street, Salem Oregon 97301

phone: 503-370-6240

fax: 503-3706379

email: jhong@willamette.edu 